International Journal of Medical Sciences
2009; 6(3):133-134

(c) Ivyspring International Publisher. All rights reserved

Short Communication

\title{
Human toxoplasmosis and the role of veterinary clinicians
}

\author{
Laura Kramer \\ Department of Animal Health- Veterinary Parasitology Laboratory- University of Parma,Parma (Italy)
}

Published: 2009.03.19

Human toxoplasmosis is one of the most common parasitic zoonoses worldwide. As for other zoonoses, veterinarians play an important role in the education of the human population regarding the risk of infection. Knowledge of the parasite's biological charateristics, life cycle and trasmission routes is necessary to correctly illustrate real risk factors to those people who have direct contact with animals (farm animals, pets, etc).

Toxoplasmosis represents one of the more difficult "educational" challenges for small animal clinicians. Veterinarians are often faced with the need to explain to their clients that it is not necessary, for example, to give away one's cat during pregnancy and that if they follow a few very simple "rules", living with their pets need not represent any risk for infection with Toxoplasma gondii.

Cats are the definitive host of T. gondii; they are the only animals that pass oocysts in their feces. They become infected by eating infected rodents, birds, or other small animals. Once oocysts are shed, they require 1 to 5 days to sporulate and become infective. Cats pass oocysts for only 2 to 3 weeks following primary infection. Mature cats are less likely to shed Toxoplasma if they have been previously infected.

Litter box hygiene is the main precaution in preventing transmission of toxoplasmosis from pet cats [1]. A Toxoplasma-infected cat that is shedding the parasite in its feces (approximately $2 \%$ of the cat population at any given time) contaminates the litter box. If the cat is allowed outside, it can contaminate the soil or water in the environment as well. Litter boxes should be cleaned daily and not placed in kitchen or dining areas. Pregnant women should avoid changing cat litter if possible. If no one else can perform the task, they should wear disposable gloves and wash their hands thoroughly with soap and wa- ter afterwards. Direct contact with cats is unlikely to result in transmission of toxoplasmosis because most cats do not leave feces on their fur for the 1 to 5 days required for oocyst sporulation. Interestingly, cat ownership has not been associated with an increase in Toxoplasma seroconversion among HIV-infected persons [2].

Along with their pregnant owners, cats should also avoid becoming infected with $T$. gondii. Therefore, cats should not be allowed to hunt and should not be fed raw or undercooked meat. This is extremely important, in that raw or undercooked meat is one of the most common routes of transmission for T. gondii, for both humans and animals [3]. Pregnant women at risk must not only avoid eating raw meat, but should also wash their hands thouroughly after handling it.

Because outdoor cats frequently defecate in gardens and T. gondii oocysts may survive for months under appropriate conditions, pregnant women should wear gloves when gardening or working with soil and should immediately wash their hands afterwards. They should also avoid eating unwashed fruits or vegetables from a garden.

Finally, because young cats more often eliminate oocysts compared to older cats, pregnant women should not adopt or handle kittens and should avoid getting a new cat during pregnancy.

A recent study [4] has shown that communication between physicians and veterinarians about zoonotic diseases is largely absent. Enhancing such communication could help prevent transmission of zoonotic agents. Links between the professions on a broader scale (e.g., through combined veterinary/medical student training and continuing education) to foster a broader consensus about zoonotic disease risks and prevention should also be encour- 
aged. This is particularly true with regards to $T$. gondii, where the consequences of infection can be very severe, while the risk of living with one's beloved cat is practically nill.

\section{References}

1. Angulo FJ, Glaser CA, Juranek DD et al. Caring for pets of immunocompromised persons. Can Vet J. 1995; 36: 217-222

2. Glaser CA, Angulo FJ, Rooney JA. Animal-associated opportunistic infections among persons infected with the human immunodeficiency virus. Clin Infect Dis.1994;18:14-24.

3. Han K, Shin DW, Lee TY, et al. Seroprevalence of Toxoplasma gondii infection and risk factors associated with seropositivity of pregnant women in Korea. J Parasitol. 2008; 94: 963-965.

4. Grant S and Olsen CW. Preventing zoonotic diseases in immunocompromised persons: the role of physicians and veterinarians. Emerg Infect Dis. 1999; 5: 159-163 